\title{
Co-targeting of EGFR by co-expressed miRNA-193a-3p/-5p in lung cancer
}

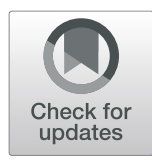

\author{
Kegan Zhu ${ }^{1 \dagger}$, Hanying Ding ${ }^{1+}, \mathrm{Ao} \mathrm{Yu}^{3+}$, Zhicong Liao ${ }^{1}$, Zheng Fu' ${ }^{1}$, Yeting Hong ${ }^{1}$, Xiaojun Wang ${ }^{2^{*}}$, \\ Chen-Yu Zhang ${ }^{1 *}$ and Xi Chen ${ }^{1 *}$
}

\begin{abstract}
MicroRNAs (miRNAs) function as important oncogenes or tumor suppressors in each stage of cancer development. While previous studies focused on the individual miRNA and its specific target gene, the cooperative functions of miRNA-3p and miRNA-5p (miRNA-3p/-5p) pairs that frequently co-exist in cells remain largely unclear. In this study, we explored the co-expression and co-targeting of the miRNA-3p/-5p pairs in lung cancer. We identified miRNA193a-3p and miRNA-193a-5p (miRNA-193a-3p/-5p) as typical co-reduced miRNA-3p/-5p pairs in NSCLC and predicted EGFR as the co-target of miRNA-193a-3p/-5p. In agreement with this, inverse expression between miRNA-193a-3p/-5p and EGFR was detected in NSCLC tissues. Furthermore, in vitro experiments validated that miRNA-193a-3p/-5p effectively suppressed migration and proliferation in lung cancer cells. Finally, data from xenograft tumor model provided in vivo evidence for miRNA-193a-3p/-5p as tumor-suppressive miRNAs by downregulating EGFR expression. In summary, our results highlight a critical role for co-expressed miRNA-193a-3p/-5p in the co-targeting of EGFR during lung tumorigenesis.
\end{abstract}

Keywords: Non-small cell lung cancer, miRNA-193a-3p, miRNA-193a-5p, Migration, Proliferation

\section{Introduction}

Lung cancer is the most common human cancer with highest morbidity and mortality, and most (80\%) cases are non-small cell lung cancer (NSCLC). NSCLC is mainly treated surgically together with chemotherapy and/or radiotherapy. A panel of well-known oncogenes drives NSCLC, including EGFR, KRAS and ALK [1]. Among all these oncogenes, EGFR (ERBB1 or HER1) is well studied. EGFR is an effective target of tyrosine kinase inhibitors (TKIs) and treatment with TKIs is the first-line therapy for patients having activating mutations. Unfortunately, these therapeutic agents have limited effects on numerous patients, which may be due to the primary and secondary drug resistance. Novel drugs, which efficiently

\footnotetext{
*Correspondence: doctorwxj326@Hotmail.com; cyzhang@nju.edu.cn; xichen@nju.edu.cn

${ }^{\dagger}$ Kegan Zhu, Hanying Ding and Ao Yu contributed equally to this work. 2Department of Thoracic Surgery, Nanjing Medical University Affilicated Cancer Hospital, Nanjing 210009, Jiangsu, China

${ }^{1}$ State Key Laboratory of Pharmaceutical Biotechnology, NJU Advanced Institute for Life Sciences (NAILS), Jiangsu Engineering Research Center for MicroRNA Biology and Biotechnology, School of Life Sciences, Nanjing University, Nanjing 210046, Jiangsu, China

Full list of author information is available at the end of the article
}

overcome and prevent resistance in NSCLC patients, are urgently needed.

In mammals, microRNAs (miRNAs), a type of small non-coding RNAs of 21-25 nt in length, bind to mRNA $3^{\prime}$-UTR and thereby inhibit protein expression or cause mRNA degradation [2]. During miRNA biogenesis, primary miRNA (pri-miRNA) is transcribed by RNA polymerase II and cleaved to 60-110 nt hairpin precursor miRNA (pre-miRNA) by Drosha. Pre-miRNA is then exported by Exportin 5 to the cytoplasm, where pre-miRNA is cleaved by Dicer to produce a double-stranded miRNA/ miRNA* duplex approximately $22 \mathrm{nt}$ in length [3, 4]. Subsequently, the functional strand, known as miRNA, is processed to mature form and followed by loading into the RNA-induced silencing complex (RISC). Meanwhile, the complementary strand is destined to be degraded [5]. However, latest studies demonstrate that miRNA* sequences can also be selected for maturation [6, 7]. Sometimes, the $5^{\prime}$ - and $3^{\prime}$ - arms of pre-miRNA can co-express and serve as different mature miRNAs. To avoid confusion, the mature miRNAs generated from the $5^{\prime}$ - and 3 '- arms of pre-miRNA are termed as miRNA-3p or miRNA-5p, respectively. Currently, there are increasing reports that

(c) The Author(s). 2019 Open Access This article is distributed under the terms of the Creative Commons Attribution 4.0 International License (http://creativecommons.org/licenses/by/4.0/), which permits unrestricted use, distribution, and 
show the co-existence of miRNA-3p and miRNA-5p (miRNA-3p/-5p) [8, 9]. The biological function of miRNA-3p/-5p co-expression deserves deeper investigation.

In this study, we investigated whether miRNA-3p/-5p possess overlapping targets and whether they work in combination to accomplish their functions. We explored co-expression and co-targeting of miRNA-3p/-5p pairs in lung cancer cells. EGFR was identified as the co-target of co-expressed miRNA-193a-3p and miRNA-193a-5p (miRNA-193a-3p/-5p). We found the direct suppression of EGFR expression by miRNA-193a-3p/-5p in vitro and identified the anti-tumor role of miRNA-193a-3p/-5p in NSCLC development in vivo.

\section{Results}

\section{Co-reduction of miRNA-193a-3p/-5p in cancer tissues}

miRNA-193a-3p/-5p were previously observed to be co-downregulated in human osteosarcoma and lung cancer $[10,11]$. To investigate the potential co-function of miRNA-3p/-5p pairs, we first selected miRNA-193a-3p/ $-5 p$ as a representative miRNA pairs and measured the expression patterns of miRNA-193a-3p/-5p in 12 pairs of NSCLC tissues and normal adjacent tissues (NATs). Both miRNA-193a-3p/-5p were consistently reduced in tumors in comparison with NATs from the same patients (Fig. 1a). The results strongly suggest concerted co-reduction of miRNA-193a-3p/-5p pairs during lung tumorigenesis.

\section{Co-targeting of EGFR by miRNA-193a-3p/-5p}

A list of co-targets of miRNA-193a-3p/-5p pairs was predicted using the RNAhybrid algorithm [12]. Although miRNA-193a-3p/-5p display no sequence homology and therefore share few predicted targets, our bioinformatics algorithm identified EGFR as a common target. The putative binding sites between miRNA-193a-3p/-5p and EGFR 3'-UTR are showed in Fig. 1b. EGFR 3'-UTR contains one binding site each for miRNA-193a-3p/-5p, and the two binding sites are non-overlapping. The minimum free energy values were -25.1 and $-28.0 \mathrm{kcal} /$ mol for hybrid between miRNA-193a-3p and EGFR and for hybrid between miRNA-193a-5p and EGFR, respectively, and the values were well within the range of free energy values associated with functional miRNA-target pairs.

Luciferase reporter assays were employed to confirm the binding ability between miRNA-193a-3p/-5p and EGFR. Mimics and antisenses were transfected into cells to overexpress and knockdown miRNA-193a-3p/-5p, respectively, and a negative control (scrambled mimic or antisense) was transfected simultaneously. As anticipated, the luciferase activity was significantly inhibited by overexpression of miRNA-193a-3p/-5p, whereas knockdown of miRNA-193a-3p/-5p increased luciferase activity (Fig. 1c). Interestingly, while reduction of luciferase activity was strengthened when miRNA-193a-3p/-5p mimics were used in combination, a reinforced luciferase activity was not detected in miRNA-193a-3p/-5p antisenses-co-transfected cells (Fig. 1c). The results implied that, although miRNA-193a-3p/-5p can co-target the EGFR transcript despite having different sequences, they have no synergistic effect on EGFR levels. Moreover, we introduced point mutations to the EGFR 3 '-UTR in the luciferase reporter plasmid. For mutated plasmid, luciferase activity was not influenced by induction of miRNA-193a-3p/-5p (Fig. 1c).

\section{Inverse relationship between miRNA-193a-3p/-5p and EGFR in cancer tissues}

We further investigated the expression pattern of EGFR in tumors to see if EGFR is negatively correlated with miRNA-193a-3p/-5p in NSCLC. We measured EGFR protein and mRNA expression levels in the same 12 pairs of NSCLC and NAT tissues. EGFR protein levels were uniformly increased in tumors (Fig. 1d), while the alteration of EGFR mRNA levels was irregular (Fig. 1e). We then explored the relationships between miRNA$193 a-3 p /-5 p$ and the EGFR protein or mRNA levels. miRNA-193a-3p/-5p had more strictly negative correlation with the EGFR protein levels than the mRNA levels in NSCLC tissues (Fig. 1f).

\section{miRNA-193a-3p/-5p co-target EGFR in vitro}

Next, we overexpressed or knocked down miRNA-193a$3 p /-5 p$ in lung cancer cells and examined the EGFR protein and mRNA levels. As expected, mimics significantly increased miRNA-193a-3p/-5p expression levels when antisenses dramatically decreased their levels in H1975 and A549 cells (Additional file 1: Figure S1A). Consequently, the EGFR protein expression were suppressed by miRNA-193a-3p/-5p mimics (Fig. 2a), whereas miRNA-193a-3p/-5p antisenses increased EGFR protein expression in cancer cells (Fig. 2b). In contrast, EGFR mRNA levels were not obviously affected by overexpression or knockdown of miRNA-193a-3p/-5p (Fig. 2c, d). Based on the results above, miRNA-193a-3p/-5p may negatively regulate EGFR expression by repressing mRNA translation. However, co-treatment of cells with both miRNA-193a-3p/-5p mimics did not enhance the suppressive effect on EGFR protein expression when compared to miRNA-193a-3p/-5p mimic alone (Fig. 2a), and the greater increase in expression of EGFR protein did not occur when miRNA-193a-3p/-5p antisenses were used simultaneously (Fig. 2b). These data imply that miRNA-193a-3p/-5p have no synergistic effect on EGFR levels.

Moreover, we constructed lentiviruses to overexpress miRNA-193a-3p/-5p and infected H1975 and A549 cells with these lentiviruses to produce functional intracellular miRNA-193a-3p/-5p. When H1975 and A549 cells were infected with lentiviruses overexpressing miRNA-193a-3p 


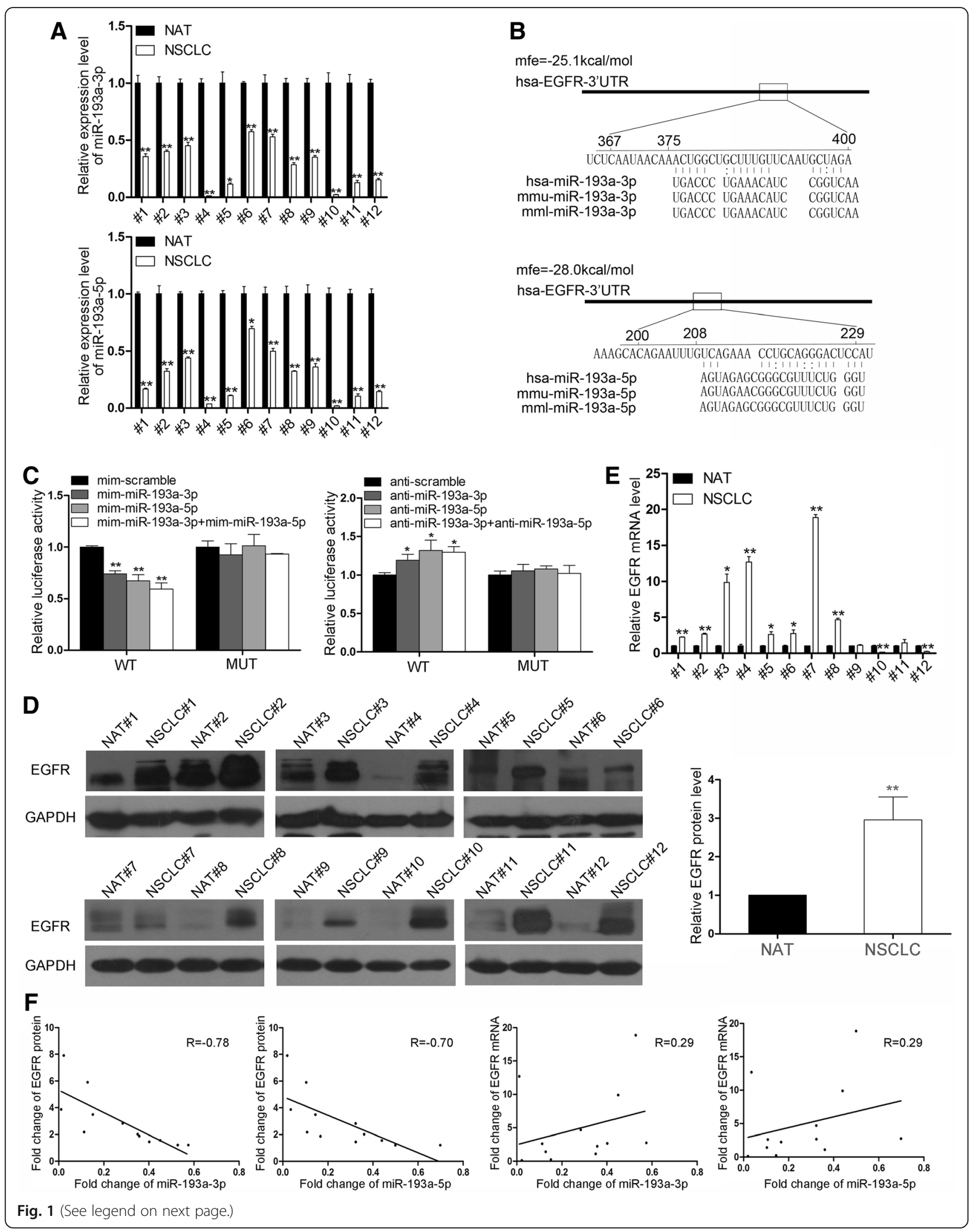


(See figure on previous page.)

Fig. 1 Inverse correlation of miRNA-193a-3p/-5p and EGFR protein expression levels in NSCLC tissues. a The relative expression levels of miRNA$193 a-3 p /-5 p$ in 12 pairs of NSCLC and NAT samples. b Schematic description of the hypothetical duplexes between miRNA-193a-3p/-5p (bottom) and EGFR mRNA (top). The predicted free energy values were indicated. The conservation of miRNAs across species, including Homo sapiens (hsa), Mus musculus ( $\mathrm{mmu}$ ) and Macaca mulatta $(\mathrm{mml})$, are displayed. $\mathbf{c}$ The relative luciferase activities that showing the binding abilities between miRNA-193a-3p/-5p and EGFR 3'UTR. WT: wild type; MUT: mutant. d EGFR protein levels in 12 pairs of NSCLC and NAT samples were analyzed by western blotting assays. Left panel: representative images; right panel: quantitative analysis. e EGFR mRNA levels in 12 pairs of NSCLC and NAT samples were analyzed by qRT-PCR. $\mathbf{f}$ The expression levels between miRNA-193a-3p/-5p and EGFR protein (left two panels) and between miRNA-193a-3p/-5p and EGFR mRNA (right two panels) in NSCLC tissues were analyzed by Pearson's correlation scatter plot. ${ }^{*} P<0.05,{ }^{*} P<0.01$

or miRNA-193a-5p, the cellular levels of miRNA-193a$3 \mathrm{p} /-5 \mathrm{p}$ were about $3-5$ fold higher than the basal levels (Additional file 1: Figure S1B). Infecting H1975 and A549 cells with miRNA-193a-3p/-5p overexpressing lentiviruses also reduced EGFR protein expression levels (Fig. 2e) but had no effect on EGFR mRNA levels (Fig. 2f) in H1975 and A549 cells. Furthermore, co-treatment with both miRNA-193a-3p/-5p overexpressing lentiviruses did not suppress the EGFR protein levels to a greater extent than either miRNA-193a-3p/-5p overexpressing lentivirus did alone (Fig. 2e). Thus, miRNA-193a-3p/-5p can co-target EGFR, but their cooperative effect is not greater than that of a single miRNA-193a-3p or miRNA-193a-5p.

\section{miRNA-193a-3p/ $-5 p$ depress the proliferation and migration of lung cancer cells}

To explore if miRNA-193a-3p/-5p have an effect on NSCLC tumorigenesis, we transfected H1975 and A549 cells with mimics and antisenses of miRNA-193a-3p/ $-5 p$ and assessed the cell migration capability by transwell assays and cell proliferation capability by CCK8 assays. Transwell assays revealed that overexpression of

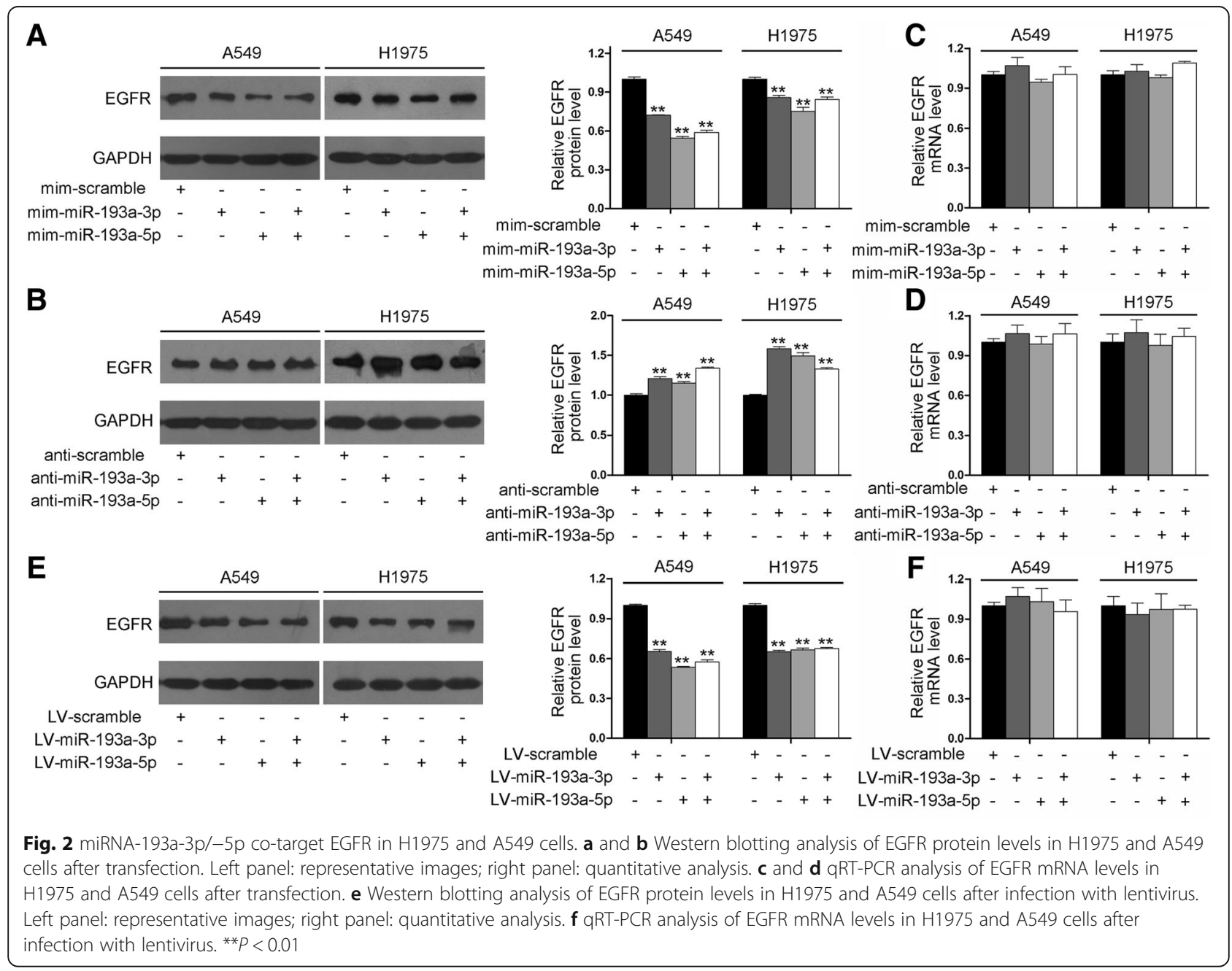


miRNA-193a-3p/-5p dramatically reduced the number of migrated H1975 and A549 cells, whereas knockdown of miRNA-193a-3p/-5p increased cell migration (Fig. 3a, b). Likewise, H1975 and A549 cells infected with miRNA-193a-3p/-5p overexpression lentivirus displayed decreased migration ability (Fig. 3c). Furthermore, overexpression of miRNA-193a-3p/-5p led to significant suppression of cell proliferation in H1975 and A549 cells, whereas miRNA-193a-3p/-5p inhibition promoted cell growth (Fig. 4a, b). Similarly, H1975 and A549 cells infected with miRNA-193a-3p/-5p overexpression lentivirus exhibited decreased proliferation ability (Fig. 4c). Overall, the data indicate that miRNA-193a-3p/-5p have negative effects on lung cancer cell proliferation/migration and therefore function as tumor suppressors during tumorigenesis. Although miRNA-193a-3p/-5p can inhibit

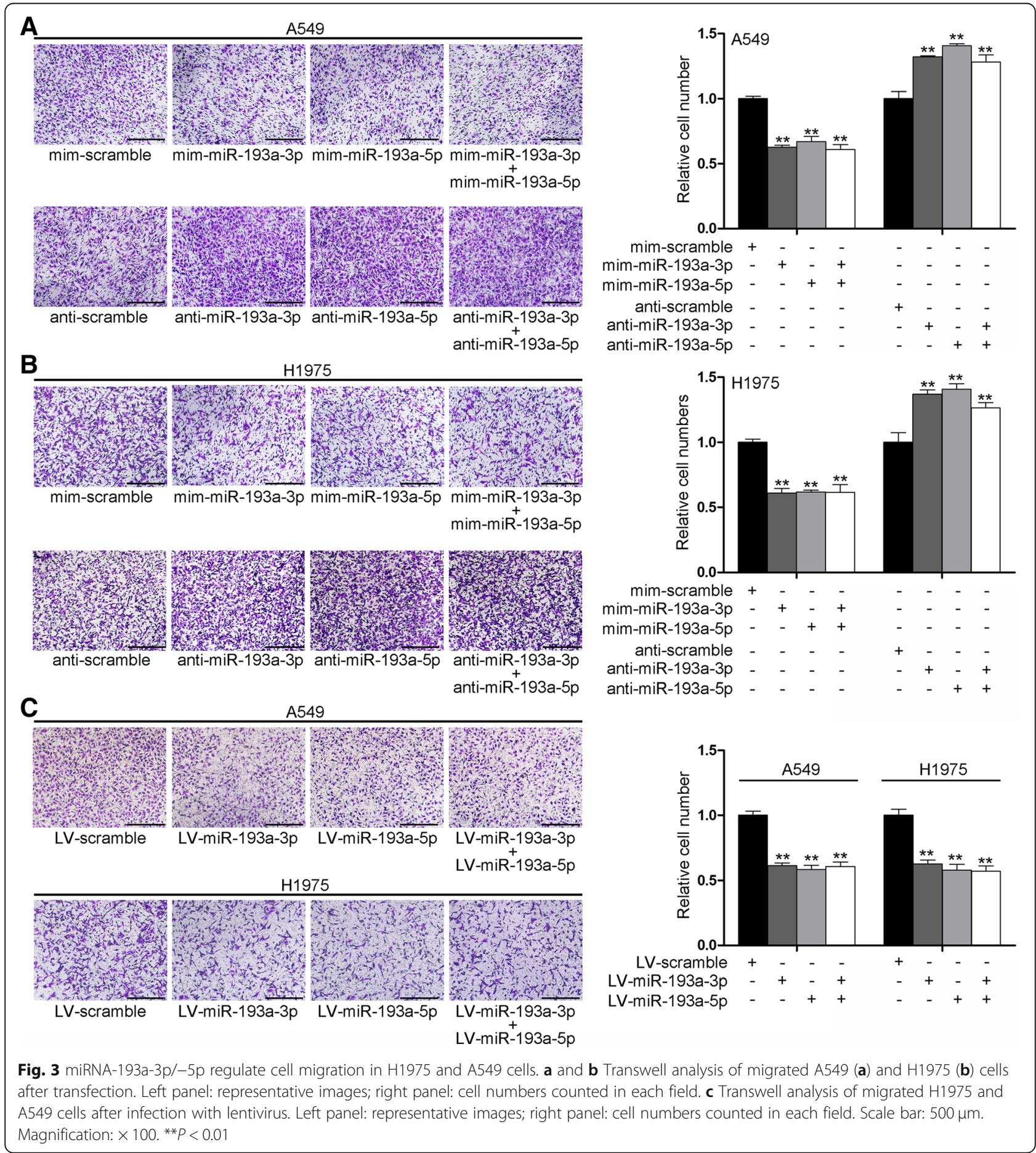




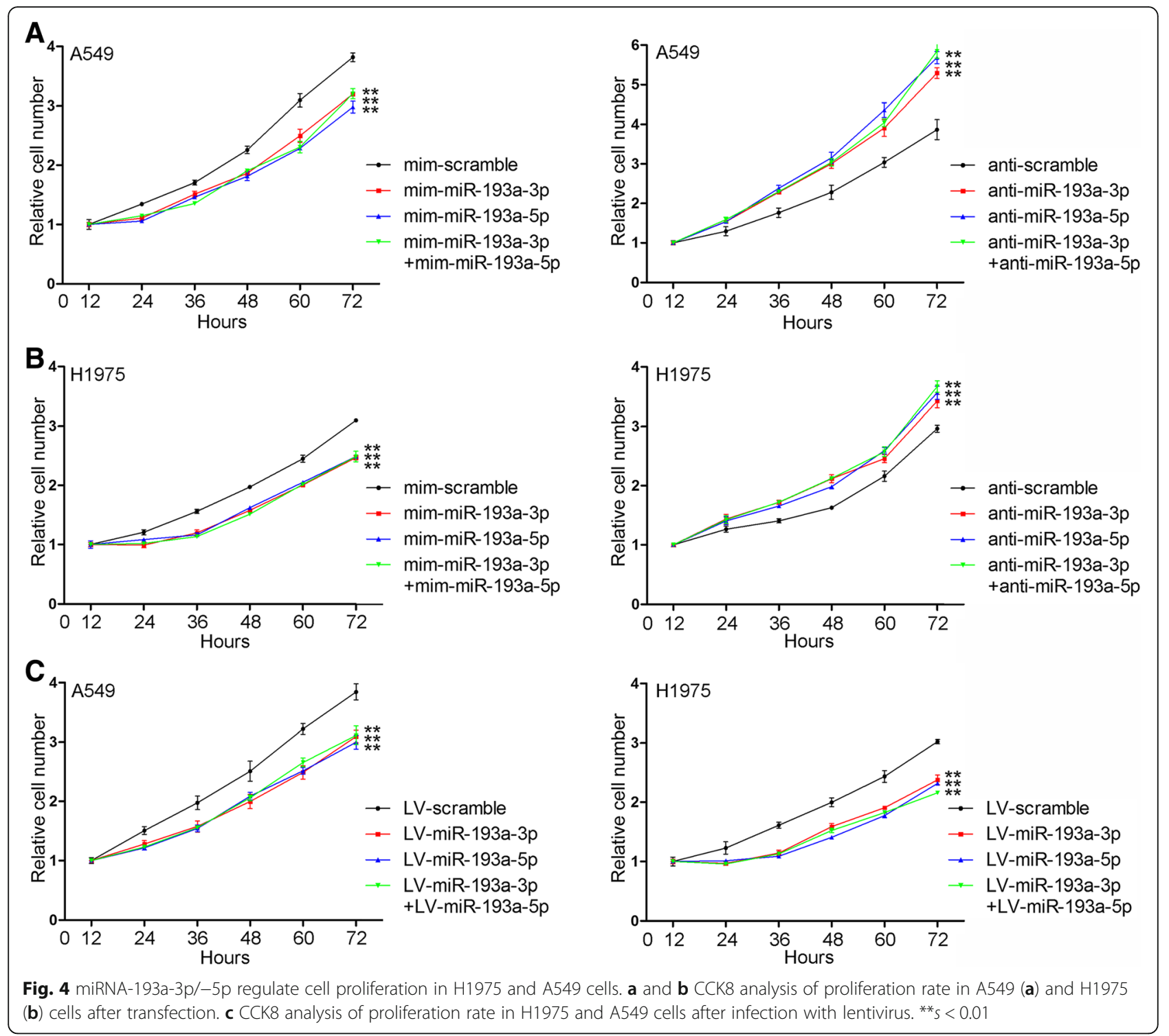

cell migration and proliferation seperately, the simultaneous overexpression of miRNA-193a-3p/-5p with mimics or lentiviruses did not show a synergistic effect on cell migration and proliferation (Figs. 3, and 4).

EGFR plays a fundamental role in each stage of tumorigenesis in many cancer types, enhancing cell growth, inhibiting cell apoptosis and contributing to angiogenesis [13-21]. For better understanding of the EGFR pathway in lung cancer, we examined the consequences of EGFR inhibition in lung cancer cells by siRNA assays. EGFR protein levels were obviously lower following treatment with EGFR siRNA compared to scrambled siRNAs in lung cancer cells (Fig. 5a). Moreover, down-expression of EGFR significantly reduced the proliferation rate in H1975 and A549 cells (Fig. 5b), suggesting that miRNA-193a-3p/-5p may inhibit cell proliferation via downregulating EGFR.
miRNA-193a-3p/-5p inhibit tumor growth in vivo by targeting EGFR

Subsequently, we investigated if miRNA-193a-3p/-5p may have an effect on tumor growth in a mouse model implanted with lung cancer cells. H1975 cells were infected with lentivirus overexpressing miRNA-193a-3p/-5p or control lentivirus and injected subcutaneously into nude mice. The miRNA-193a-3p/-5p expression levels in H1975 cells after lentiviral infection were determined (Additional file 1: Figure S1B). Compared to the control group, the tumors in miRNA-193a-3p or miRNA-193a-5p-overexpressing group grew much faster (Fig. 6a). Mice were then sacrificed and tumor size/weight were measured. As expected, tumors from the miRNA-193a-3p or miRNA193a-5p-overexpressing group were lighter and smaller (Fig. 6b-d). Tumors from the miRNA-193a-3p or miRNA-193a-5p-overexpressing group had an increase 


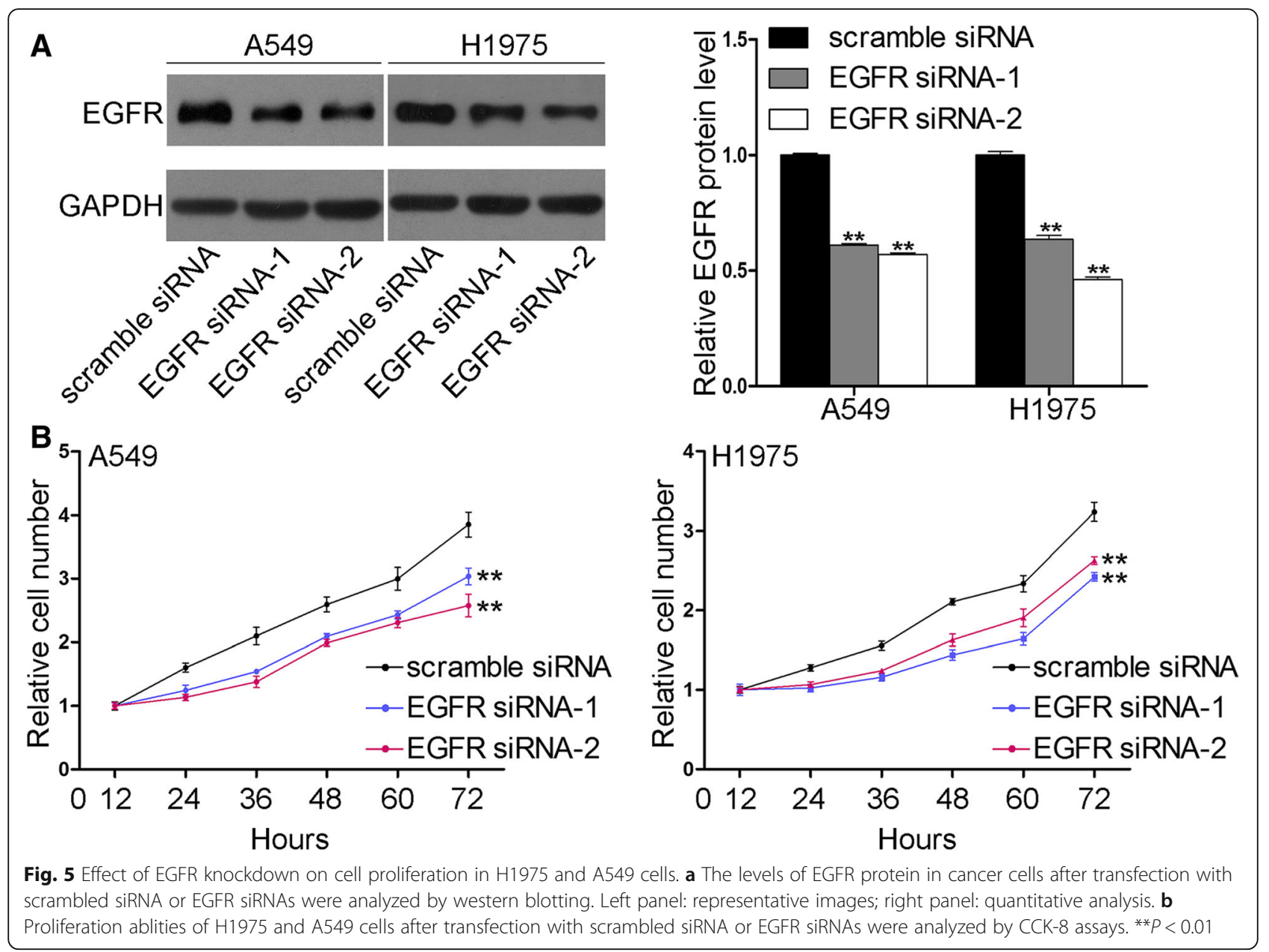

in miRNA expression (Fig. 6e) and decrease in EGFR protein expression levels in tumors (Fig. 6f). Finally, we performed immunohistochemical staining assays to examine the PCNA and Ki-67 levels. The staining intensity of Ki-67 and PCNA was decreased in tumors from the miRNA-193a-3p or miRNA-193a-5p-overexpressing group (Fig. $6 \mathrm{~g}$ ), which means the proliferative ability of cancer cells was depressed. These data offer in vivo evidence for the inhibitory effect of miRNA-193a-3p/-5p on lung cancer through the inhibition of EGFR expression.

\section{Discussion}

Current research in tumor biology has identified several new prognostic and predictive biomarkers and therapeutic targets for NSCLC and uncovered some dominant oncogenes involved in NSCLC carcinogenesis [22]. EGFR is one of such gene, whose central contribution to the deregulation of NSCLC cell behavior has become clear. During last decades, miRNAs are proved to play important roles in cancers by targeting oncogenes or tumor suppressors. In lung cancer, miR-193a-3p have been demonstrated to regulate ERBB4, KRAS and S6K2, when
miR-193a-5p play roles in regulating WT1-E-cadherin axis, mTOR and PIK3R3 [10, 23, 24]. Herein we showed that miRNA-193a-3p/-5p behaved together as tumor-suppressive miRNAs in vitro and in vivo and proved that EGFR is a key target through which miRNA-193a-3p/-5p contribute to cancer development.

Chemotherapy and/or radiotherapy are the main therapeutic agents for NSCLC for decades [25-27]. At present, several targeted drugs have significantly altered the landscape of NSCLC therapy. However, there are still lots of patients that have no response to these therapies or become resistance to treatment. Hence, it remains important to overcome the problems of drug resistance and develop new therapeutic strategies. Considering that miRNA-193a-3p/-5p are upstream regulators of EGFR, it could be possible to upregulate miRNA-193a-3p/-5p for containment of EGFR during tumorigenesis. To date, increasing evidences demonstrated that miRNAs are very promising therapeutic molecules for human cancers $[28,29]$. Here we suggest that the replacement treatment with miRNA-193a-3p/-5p may be a potential solution for NSCLC having miRNA-193a-3p/-5p reduction. 


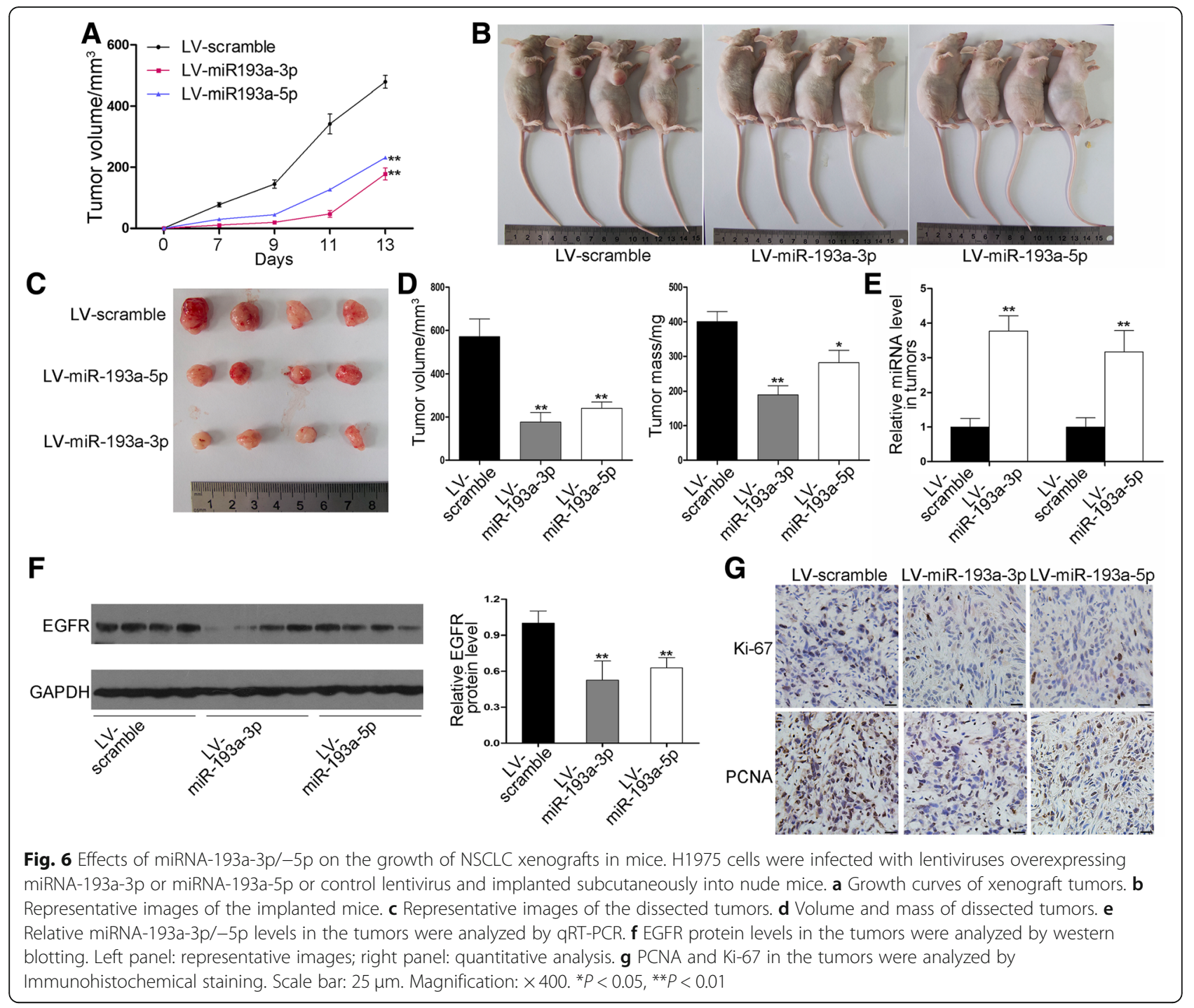

More studies are necessary to explore the possibility of targeting miRNA-193a-3p/-5p for NSCLC therapy and to develop feasible miRNA delivery systems.

During miRNA biogenesis, the same pre-miRNA may generate two miRNA species from the $5^{\prime}$ and $3^{\prime}$ arms. In traditional thought, one miRNA species remains while the other one is degraded. However, more researches have proved the coexistence of two mature miRNA species in cells, albeit in different concentrations. Despite the widespread evidence for the consistency of expression of miRNA-3p/ $-5 p$ pairs, the reason for this co-expression is not fully understood. However, most studies about miRNAs to date focused on the regulation of a specific gene by a specific miRNA, and the overall cellular functions affected by co-expressed miRNA-3p/-5p pairs remain unknown. Considering the current incomplete understanding of miRNA-3p/ $-5 p$ pairs and their common targets in cancer, more studies on the biological roles of miRNA-3p/-5p pairs are urgently needed. In the present study, bioinformatic analysis of EGFR 3'-UTR identified two non-overlapping binding sites for miRNA-193a-3p/-5p. In vitro and in vivo data supported the hypothesis of co-targeting of EGFR by miRNA-193a-3p/-5p. Because there are currently very few reports of miRNA-3p/-5p pairs targeting the same genes $[10,11,30]$, this study offers new evidence that miRNA-3p/ $-5 p$ can simultaneously repress a same gene. Although we did not observe a cooperative and synergistic effect of miRNA-193a-3p/-5p on EGFR repression and identify more efficient and potent co-regulation of target gene by miRNA- $3 p /-5 p$ pairs than by a single miRNA, we propose that miRNA-193a-3p/-5p may work in concert to provide a fail-proof mode to make sure that when a miRNA-3p/miRNA-5p species is not functional, the other one is still functional. In summary, our results suggest the importance of further elucidating possible cooperation of co-expressed miRNA-3p/-5p pairs in cancer pathogenesis. 
In summary, this study demonstrates that miRNA$193 a-3 p /-5 p$ possess tumor-suppressive ability and could inhibit NSCLC carcinogenesis through downregulating EGFR. Co-downregulation of miRNA-193a$3 p /-5 p$ may be a reason for the dysregulation of EGFR function in lung cancer. In future, miRNA-193a-3p/-5p may provide a strategy towards EGFR-targeted lung cancer therapy, especially for those patients with drug resistance.

\section{Conclusion}

This study highlights an important role for co-expressed miR-193a-3p/-5p in the regulation of EGFR in lung cancer cells. miR-193a-3p/-5p may act as novel anti-cancer drug that have clinical transformation values in the EGFR-targeted therapy.

\section{Materials and methods}

\section{Human tissues}

Paired NSCLC and NAT tissue samples were derived from the Nanjing Drum Tower Hospital Affiliated to Medical School of Nanjing University (Nanjing, China). All patients involved signed an informed consent form. This study was approved by the Ethics Committee of Nanjing University. Additional file 1: Table S1 summarized the patient information.

\section{Cell lines and reagents}

A549 and H1975 lung cancer cell lines were purchased from the Shanghai Institute of Cell Biology, Chinese Academy of Sciences (Shanghai, China). Anti-EGFR antibody was purchased from R\&D systems (USA, 1:1000). Anti-GAPDH antibody was purchased from Santa Cruz Biotechnology (CA, USA, 1:2000).

\section{miRNA target prediction}

The co-targets of the miRNA-193a-3p/-5p pairs were predicted using the RNAhybrid algorithm [12]. First, only the putative targets shared between miRNA-193a-3p/-5p were selected. Second, hybrid energy should be lower than $-25 \mathrm{kcal} / \mathrm{mol}$. Third, there should be interspecies conservation within the miRNA binding sites. Based on these rules, approximately 100 genes were identified as candidate co-targets of miRNA-193a-3p/-5p pairs.

\section{Quantitative RT-PCR (qRT-PCR) assay}

Total RNA was extracted using TRIzol Reagent (Invitrogen) according to the manufacturer's instructions. miRNA detection was performed with Taqman probes (Applied Biosystems) according to the manufacturer's instructions. U6 snRNA served as the internal control of miRNAs because U6 is a common reference gene in analyzing miRNAs with qRT-PCR assays [31-33]. The relative amount of miRNA expression was calculated with the eq. $2^{-\Delta \Delta C T}$, in which $\Delta \Delta \mathrm{C}_{\mathrm{T}}=\left(\mathrm{C}_{\mathrm{T}}\right.$ miRNA $-\mathrm{C}_{\mathrm{T}}$ U6 $)$ test condition $\left(\mathrm{C}_{\mathrm{T}}\right.$ miRNA $-\mathrm{C}_{\mathrm{T}}$ U6 $)$ control condition. EGFR mRNA expression levels were determined using the SYBR Green (Invitrogen) method and were normalized to GAPDH. Primers were as follows: 5'-CGAGCCACA TCGCTCAGACA-3' (GAPDH, sense); 5'-GTGGTGA AGACGCCAGTGGA-3' (GAPDH, antisense); 5' -TTG CCGCAAAGTGTGTAACG-3' (EGFR, sense); 5'-GT CACCCCTAAATGCCACCG-3' (EGFR, antisense).

\section{Cell transfection}

EGFR siRNAs and miRNA mimics/antisenses were synthesized by Genepharma (Shanghai, China). Cells (in 6-well plate) were transfected with Lipofectamine 2000 (Invitrogen) when they grew to $70 \%$ confluence. For each well, $100 \mathrm{pmol}$ of mimics, antisenses or siRNAs were used. For co-transfection of miRNA-193a-3p/-5p mimics or antisenses, $50 \mathrm{pmol}$ of each miRNA were used. After $48 \mathrm{~h}$, cells were harvested for western blotting and qRT-PCR analysis. SiRNA sequences were: EGFR SiRNA-1: 5'-GCAGUCUUAUCUAACUAUG AUGCAA-3'; EGFR siRNA-2: 5'-GCAGUGACU UUCUCAGCAA-3'.

\section{Cell infection}

Scrambled lentivirus and lentivirus overexpressing miRNA-193a-3p or miRNA-193a-5p were constructed by Realgene (Nanjing, China). Lentivirus was added to H1975 and A549 cells at an MOI of 3. Cells were harvested for further investigation $48 \mathrm{~h}$ post-infection.

\section{Luciferase assay}

A 501-bp fragment of the 3'UTR of EGFR Transcript Variant 4, containing the predicted miRNA-193a-3p/-5p binding sites, was amplified and inserted into a p-MIRreport plasmid (Ambion, Austin, TX, USA). Mutant 3'UTR of EGFR Transcript Variant 4 was synthesized and inserted into the same plasmid. The mutant sequences were 396 to 399 for miRNA-193a-3p and 225 to 229 for miRNA-193a-5p. For luciferase assays, 40 pmol of miRNA mimics or scrambled mimics were transfected into 12 -well plates along with $0.8 \mu \mathrm{g}$ of reporter plasmid and $0.8 \mu \mathrm{g}$ of transfection control $\beta$-galactosidase plasmid (Ambion). Luciferase activity was measured with a kit (Promega, Madison, WI, USA) 24 h post-transfection.

\section{Cell proliferation assay}

The cell proliferation ability is verified by CCK-8 assays. For each well of 96-well plates, $5 \times 10^{3}$ cells were seeded. Cell proliferation index was assessed at 12, 24, 36, 48, 60 and $72 \mathrm{~h}$. 


\section{Cell migration assay}

In migration assay, an $8.0 \mu \mathrm{m}$ Pore Polycarbonate Membranes (Corning, New York, USA) was placed in 24-wells. Then $3 \times 10^{4}$ cells in $100 \mu \mathrm{l}$ serum-free medium were added to the upper chamber, and 10\% FBS-containing medium was added to the lower chamber. Cells were incubated for another $12 \mathrm{~h}$ for A549 cells or $24 \mathrm{~h}$ for H1975 cells at $37^{\circ} \mathrm{C}$. Cells that migrated from the upper chamber to the bottom of the membrane were fixed with $4 \%$ paraformaldehyde, stained with $0.1 \%$ crystal violet and visualized under a microscope.

\section{Establishment of tumor xenografts in nude mice}

Nude mice were purchased from the Model Animal Research Center of Nanjing University (Nanjing, China). All animal care and handling procedures were performed in accordance with the National Institutes of Health's Guide for the Care and Use of Laboratory Animals. H1975 cells were infected with control lentivirus (LV-scramble) or lentiviruses to express miRNA-193a-3p or miRNA-193a-5p, and $5 \times 10^{6} \mathrm{H} 1975$ cells were injected subcutaneously into the mice (3 groups, 10 mice/group). Tumor widths and lengths were measured every 2 days. Then the mice were sacrificed and the xenograft tumors were photographed and weighed. Total RNA and protein were isolated from the tumors for further analysis, and the xenograft tumors were fixed in $4 \%$ paraformaldehyde followed by immunohistochemical staining of Ki-67 and PCNA. Images were obtained using the microscope at $\times 400$ magnification (Olympus, Japan).

\section{Statistical analyses}

Each assay was repeated independently for at least three times. Data were presented as the mean $\pm \mathrm{SE}$. The differences were considered statistically significant at $P<0.05$ (Student's t-test). * indicates $P<0.05$; * indicates $P<0.01$.

\section{Additional file}

Additional file 1: Figure S1. The expression levels of miR-193a-3p and $-5 p$ in A549 and H1975 cells after transfection or infection. Table S1. Clinical features of lung cancer patients. (DOCX $202 \mathrm{~kb}$ )

\section{Abbreviations}

3'-UTR: 3'-untranslated region; ALK: Anaplastic lymphoma kinase; EGFR: Epidermal growth factor receptor; KRAS: the V-Ki-ras2 Kirsten rat sarcoma viral oncogene homolog; miRNAs: microRNAs; NSCLC: Non-small cell lung cancer; TKIs: Tyrosine kinase inhibitors

\section{Acknowledgements}

Not applicable.

\section{Funding}

This work was supported by grants from the National Basic Research Program of China (973 Program) (No. 2014CB542300), the National Natural Science Foundation of China (No. 31741075) and the Natural Science Foundation of Jiangsu Province (No. BE2016737).

\section{Availability of data and materials}

All data generated or analysed during this study are included in this published article and its Additional file 1.

\section{Authors' contributions}

$X C$ and $C Y Z$ formulated the hypothesis, commented on this study and edited the manuscript. $K Z$ and $H D$ performed the experiments and $A Y, Z L$, $Z F$, YH offered some assistance. $K Z$ did data analysis and $X W$ offered some assistance. All authors reviewed the manuscript. All authors read and approved the final manuscript.

\section{Ethics approval and consent to participate}

This study was approved by the Ethics Committee of Nanjing University. All animal care and handling procedures were performed in accordance with the National Institutes of Health's Guide for the Care and Use of Laboratory Animals and were approved by the Institutional Review Board of Nanjing University.

Consent for publication

Not applicable.

\section{Competing interests}

The authors declare that they have no competing interests.

\section{Publisher's Note}

Springer Nature remains neutral with regard to jurisdictional claims in published maps and institutional affiliations.

\section{Author details}

${ }^{1}$ State Key Laboratory of Pharmaceutical Biotechnology, NJU Advanced Institute for Life Sciences (NAILS), Jiangsu Engineering Research Center for MicroRNA Biology and Biotechnology, School of Life Sciences, Nanjing University, Nanjing 210046, Jiangsu, China. ${ }^{2}$ Department of Thoracic Surgery, Nanjing Medical University Affilicated Cancer Hospital, Nanjing 210009, Jiangsu, China. ${ }^{3}$ Department of Cardio-Thoracic Surgery, Nanjing Drum Tower Hospital Affiliated to Medical School of Nanjing University and Nanjing Multi-Center Biobank, Nanjing 210093, Jiangsu, China.

Received: 27 December 2017 Accepted: 14 March 2019

Published online: 06 December 2019

\section{References}

1. Gasparini P, Cascione L, Landi L, Carasi S, Lovat F, Tibaldi C, Ali G, D'Incecco A, Minuti G, Chella A, et al. microRNA classifiers are powerful diagnostic prognostic tools in ALK-, EGFR-, and KRAS-driven lung cancers. P Natl Acad Sci USA. 2015:112(48):14924-9.

2. Lee YS, Nakahara K, Pham JW, Kim K, He Z, Sontheimer EJ, Carthew RW. Distinct roles for Drosophila Dicer-1 and Dicer-2 in the siRNA/miRNA silencing pathways. Cell. 2004;117(1):69-81.

3. Bartel DP. MicroRNAs: genomics, biogenesis, mechanism, and function. Cell. 2004;116(2):281-97.

4. Winter J, Jung S, Keller S, Gregory Rl, Diederichs S. Many roads to maturity: microRNA biogenesis pathways and their regulation. Nat Cell Biol. 2009; 11(3):228-34.

5. O'Toole AS, Miller S, Haines N, Zink MC, Serra MJ. Comprehensive thermodynamic analysis of 3 ' double-nucleotide overhangs neighboring Watson-crick terminal base pairs. Nucleic Acids Res. 2006:34(11):3338-44.

6. Yang JS, Phillips MD, Betel D, Mu P, Ventura A, Siepel AC, Chen KC, Lai EC. Widespread regulatory activity of vertebrate microRNA* species. Rna. 2011; 17(2):312-26.

7. Ro S, Park C, Young D, Sanders KM, Yan W. Tissue-dependent paired expression of miRNAs. Nucleic Acids Res. 2007;35(17):5944-53.

8. Choo KB, Soon YL, Nguyen PNN, Hiew MSY, Huang CJ. MicroRNA-5p and-3p co-expression and cross-targeting in colon cancer cells. J Biomed Sci. 2014; 21:95

9. Jiang LL, Huang Q, Zhang SY, Zhang QF, Chang JH, Qiu XS, Wang EH. HsamiR-125a-3p and hsa-miR-125a-5p are downregulated in non-small cell lung cancer and have inverse effects on invasion and migration of lung cancer cells. BMC Cancer. 2010;10:318.

10. Yu T, Li J, Yan M, Liu L, Lin H, Zhao F, Sun L, Zhang Y, Cui Y, Zhang F, et al. MicroRNA-193a-3p and -5p suppress the metastasis of human non-small- 
cell lung cancer by downregulating the ERBB4/PIK3R3/mTOR/S6K2 signaling pathway. Oncogene. 2015;34(4):413-23.

11. Pu Y, Zhao F, Cai W, Meng X, Li Y, Cai S. MiR-193a-3p and miR-193a-5p suppress the metastasis of human osteosarcoma cells by down-regulating Rab27B and SRR, respectively. Clin. Exp. Metastasis. 2016;33(4):359-72.

12. Rehmsmeier $M$, Steffen $P$, Hochsmann $M$, Giegerich R. Fast and effective prediction of microRNA/target duplexes. Rna. 2004;10(10):1507-17.

13. Zhao $L$, Jiang $X$, Peng F, Zhou R. correlation between epidermal growth factor receptor (EGFR) intron1 (CA) n microsatellite polymorphism, lymph node metastasis and expression of EGFR in non-small cell lung cancer Zhonghua Bing Li Xue Za Zhi. 2010;39(7):476-7.

14. McMillen $E$, Ye F, Li G, Wu Y, Yin G, Liu W. Epidermal growth factor receptor (EGFR) mutation and p-EGFR expression in resected non-small cell lung cancer. Exp Lung Res. 2010;36(9):531-7.

15. Xu L, Hausmann M, Dietmaier W, Kellermeier S, Pesch T, Stieber-Gunckel M, Lippert E, Klebl F, Rogler G. Expression of growth factor receptors and targeting of EGFR in cholangiocarcinoma cell lines. BMC Cancer. 2010;10:302.

16. Schunemann DP, Grivicich I, Regner A, Leal LF, de Araujo DR, Jotz GP, Fedrigo CA, Simon D, da Rocha AB. Glutamate promotes cell growth by EGFR signaling on U-87MG human glioblastoma cell line. Pathol Oncol Res. 2010;16(2):285-93.

17. Okamoto K, Okamoto I, Okamoto W, Tanaka K, Takezawa K, Kuwata K, Yamaguchi H, Nishio K, Nakagawa K. Role of survivin in EGFR inhibitorinduced apoptosis in non-small cell lung cancers positive for EGFR mutations. Cancer Res. 2010;70(24):10402-10.

18. Li Z, Zhou S, Zhang L, Su C, Hang J, Zhao Y, Su B, Zhou C. BIM induction of apoptosis triggered by EGFR-sensitive and resistance cell lines of non-smallcell lung cancer. Med Oncol. 2011;28(2):572-7.

19. Thoennissen NH, O'Kelly J, Lu D, Iwanski GB, La DT, Abbassi S, Leiter A, Karlan B, Mehta R, Koeffler HP. Capsaicin causes cell-cycle arrest and apoptosis in ER-positive and -negative breast cancer cells by modulating the EGFR/HER-2 pathway. Oncogene. 2010;29(2):285-96.

20. Kanashiro CA, Schally AV, Zarandi M, Hammann BD, Varga JL. Alterations of EGFR/HER, angiogenesis and apoptosis pathways after therapy with antagonists of growth hormone releasing hormone and bombesin in nonsmall cell lung cancer. Int J Oncol. 2007;30(4):1019-28.

21. Meert AP, Feoli F, Martin B, Ninane V, Sculier JP. Angiogenesis in preinvasive, early invasive bronchial lesions and micropapillomatosis and correlation with EGFR expression. Histopathology. 2007;50(3):311-7.

22. Hensing T, Chawla A, Batra R, Salgia R. A personalized treatment for lung Cancer: molecular pathways, targeted therapies, and genomic characterization. Adv Exp Med Biol. 2014;799:85-117.

23. Chen J, Gao S, Wang C, Wang Z, Zhang H, Huang K, Zhou B, Li H, Yu Z, Wu $J$, et al. Pathologically decreased expression of miR-193a contributes to metastasis by targeting WT1-E-cadherin axis in non-small cell lung cancers. J Exp Clin Cancer Res. 2016;35(1):173.

24. Fan Q, Hu X, Zhang H, Wang S, Zhang H, You C, Zhang CY, Liang H, Chen $X, B a$ Y. MiR-193a-3p is an important tumour suppressor in lung Cancer and directly targets KRAS. Cell Physiol Biochem. 2017;44(4):1311-24.

25. Amit D, Tamir S, Birman T, Gofrit ON, Hochberg A. Development of targeted therapy for bladder cancer mediated by a double promoter plasmid expressing diphtheria toxin under the control of IGF2-P3 and IGF2-P4 regulatory sequences. Int J Clin Exp Med. 2011;4(2):91-102.

26. Amit D, Hochberg A. Development of targeted therapy for bladder cancer mediated by a double promoter plasmid expressing diphtheria toxin under the control of H19 and IGF2-P4 regulatory sequences. J TransI Med. 2010;8:134.

27. Manyam BV, Mallick IH, Abdel-Wahab MM, Reddy CA, Remzi FH, Kalady MF, Lavery I, Koyfman SA. The impact of preoperative radiation therapy on Locoregional recurrence in patients with stage IV rectal Cancer treated with definitive surgical resection and contemporary chemotherapy. J Gastrointest Surg. 2015;19(9):1676-83.

28. Yin Y, Cai $X$, Chen $X$, Liang HW, Zhang YJ, Li J, Wang ZY, Chen XL, Zhang W, Yokoyama S, et al. Tumor-secreted miR-214 induces regulatory $T$ cells: a major link between immune evasion and tumor growth. Cell Res. 2014; 24(10):1164-80.

29. Zhang YJ, Liu DQ, Chen X, Li J, Li LM, Bian Z, Sun F, Lu JW, Yin YA, Cai X, et al. Secreted Monocytic miR-150 enhances targeted endothelial cell migration. Mol Cell. 2010;39(1):133-44.

30. Mitra R, Lin CC, Eischen CM, Bandyopadhyay S, Zhao Z. Concordant dysregulation of miR-5p and miR-3p arms of the same precursor microRNA may be a mechanism in inducing cell proliferation and tumorigenesis: a lung cancer study. RNA. 2015;21(6):1055-65.
31. Chen X, Ba Y, Ma L, Cai X, Yin Y, Wang K, Guo J, Zhang Y, Chen J, Guo X, et al. Characterization of microRNAs in serum: a novel class of biomarkers for diagnosis of cancer and other diseases. Cell Res. 2008;18(10):997-1006.

32. Zhang H, Bai M, Deng T, Liu R, Wang X, Qu Y, Duan J, Zhang L, Ning T, Ge $\mathrm{S}$, et al. Cell-derived microvesicles mediate the delivery of miR-29a/c to suppress angiogenesis in gastric carcinoma. Cancer Lett. 2016;375(2):331-9.

33. Adam L, Wszolek MF, Liu CG, Jing W, Diao L, Zien A, Zhang JD, Jackson D, Dinney CP. Plasma microRNA profiles for bladder cancer detection. Urol Oncol. 2013;31(8):1701-8.

\section{Ready to submit your research? Choose BMC and benefit from:}

- fast, convenient online submission

- thorough peer review by experienced researchers in your field

- rapid publication on acceptance

- support for research data, including large and complex data types

- gold Open Access which fosters wider collaboration and increased citations

- maximum visibility for your research: over $100 \mathrm{M}$ website views per year

At BMC, research is always in progress.

Learn more biomedcentral.com/submissions 